\title{
Intervenção Breve para Redução do Consumo de Álcool e suas Consequências em Estudantes Universitários Brasileiros
}

\author{
Brief Intervention to Reduce Alcohol Consumption and its Consequences \\ in Brazilian University Students
}

\author{
Érika Correia Silva* \& Adriana Marcassa Tucci \\ Universidade Federal de São Paulo, Santos, SP, Brasil
}

\begin{abstract}
Resumo
O aumento do consumo de álcool entre universitários tem gerado preocupações nos estudiosos da área que buscam intervenções preventivas eficazes à diminuição desse consumo. Este estudo avaliou o padrão de consumo de álcool e suas consequências, antes e após a aplicação da intervenção BASICS (Brief Alcohol Screnning and Intervention for College Students) em uma amostra de universitários brasileiros, sendo em sua maioria estudantes do sexo feminino $(53,1 \%)$ e da faixa etária de 18 a 24 anos (84,4\%). Foram utilizados os instrumentos The Alcohol Use Disorders Identification Test (AUDIT) e o Rutgers Alcohol Problem Index (RAPI) para caracterização do consumo de álcool e levantamento de suas consequências. Os que pontuaram acima de oito no AUDIT foram convidados a participar de um grupo experimental ou controle. A análise dos dados revelou que não houve diferença estatisticamente significativa entre os grupos após a intervenção. Em ambos os grupos houve: diminuição das doses consumidas, menos prática de binge drinking e redução de algumas das consequências negativas. Tal resultado indica uma importante contribuição na área e sugere que o levantamento do padrão de consumo de álcool e o seu respectivo feedback pode gerar efeitos positivos na diminuição do consumo de álcool, assim como das suas consequências entre estudantes universitários.

Palavras-chave: Estudantes universitários, abuso de álcool, intervenção precoce, prevenção.
\end{abstract}

\begin{abstract}
The increase in alcohol consumption among college students has increased concerns among researchers seeking effective preventive interventions to decrease their consumption. This study assessed the pattern of alcohol consumption and its consequences before and after applying the BASICS intervention (Brief Alcohol Screnning and Intervention for College Students) in a sample of Brazilian university, with mostly female students (53.1\%) aged between 18 and 24 years (84.4\%). The Alcohol Use Disorders Identification Test (AUDIT) and Rutgers Alcohol Problem Index (RAPI) tools were used to characterize alcohol consumption and its consequences. Those who scored above eight on the AUDIT were invited to participate in an experimental or control group. Data analysis revealed no statistically significant difference between groups. In both groups there were: a decrease in used dosages, less practice of binge drinking, and decrease in some negative consequences. This result indicates an important contribution in the area and suggests that the screening of alcohol consumption pattern and its respective feedback can generate positive effects in the reduction of alcohol consumption, as well as its consequences among college students.

Keywords: College students, alcohol abuse, early intervention, prevention.
\end{abstract}

O ensino superior no Brasil tem se expandido de forma considerável, com destaque para o Programa de Apoio a Planos de Reestruturação e Expansão das Universidades Federais (REUNI), instituído pelo Decreto Lei n ${ }^{\circ} 6.096$ de 24 de Abril de 2007, que vem ampliando o acesso e permanência dos estudantes no ensino superior (Cislaghi, 2010).

A criação do REUNI proporcionou um aumento no número de vagas e de cursos oferecidos, além de trazer

\footnotetext{
${ }^{*}$ Endereço para correspondência: Universidade Federal de São Paulo, Rua Silva Jardim, 136, Vila Mathias, Santos, SP, Brasil, 11015-020. E-mail: eri_correia@ hotmail.com e atucci@unifesp.br
}

novas perspectivas para o ensino superior, com modificação nas políticas de ingresso, na estrutura acadêmica, inclusão e assistência aos estudantes durante sua formação (Cislaghi, 2010).

$\mathrm{O}$ ingresso no ensino superior representa uma fase de mudanças e desafios aos estudantes. São mudanças no convívio social e nas atividades cotidianas que geram maior autonomia e liberdade ao estudante. No entanto, essa é uma fase que pode levar à aquisição de comportamentos pouco adequados e muitas vezes associados a um consumo excessivo de bebidas alcoólicas (Haas, Smith, Kagan, \& Jacob, 2012; Lorant, Nicaise, Soto, \& D'Hoore, 2013; Ramis et al., 2012). 
Silva, E. C. \& Tucci, A. M. (2015). Intervenção Breve para Redução do Consumo de Álcool e suas Consequências em Estudantes Universitários Brasileiros.

Segundo dados do I Levantamento Nacional sobre o Uso de Álcool, Tabaco e Outras Drogas entre Universitários das 27 Capitais Brasileiras (Andrade, Duarte, \& Oliveira, 2010) e de estudos como o de Grossbard et al. (2010) e de Silva e Petroski (2012), essa fase tem sido considerada crítica, com maior vulnerabilidade para o início e manutenção do consumo de álcool.

Segundo dados da Organização das Nações Unidas (ONU, 2013), o álcool é a substância psicoativa mais consumida no mundo, com crescimento expressivo do consumo entre os estudantes universitários. $\mathrm{O}$ aumento desse consumo nessa população tem gerado preocupações nos estudiosos da área e, em decorrência disto, estudos têm avaliado o padrão deste consumo, assim como intervenções preventivas eficazes que o diminuam (Lorant et al., 2013; Pedrosa, Camacho, Lambert, \& Oliveira, 2011; Ramis et al., 2012; Wagner et al., 2012).

A partir da identificação da necessidade de diminuição do consumo do álcool, as intervenções breves, com técnicas focais e de curta duração têm se tornado uma forma de reduzir os danos decorrentes do consumo de álcool (Marques \& Furtado, 2004).

A intervenção breve foi inicialmente proposta como uma abordagem terapêutica para dependentes de álcool por Sanchez-Craig, em 1972 (citado por Marques e Furtado, 2004). Atualmente, essas intervenções apresentam estratégias e técnicas diferenciadas que podem variar com relação a suas metas, duração e profissional que desenvolverá a técnica, além de ser direcionada por referenciais teóricos específicos, mas em sua maioria originários das teorias comportamental e cognitivista (Marques \& Furtado, 2004).

Todas as intervenções breves envolvem estratégias educacionais, de aconselhamento breve e entrevista motivacional e têm como pressupostos teóricos que o comportamento disfuncional pode ser modificado (Difulvio, Linowski, Mazziotti, \& Puleo, 2012; Dimeff, Baer, Kivlahan, \& Marlatt, 2002; Marques \& Furtado, 2004).

Dimeff et al. (2002) desenvolveram o Brief Alcohol Screnning and Intervention for College Students (BASICS), a única intervenção breve citada na literatura que foi desenvolvida e padronizada especificamente para estudantes universitários. Baseada nos pressupostos da redução de danos, essa intervenção tem como objetivo reduzir o consumo excessivo de álcool por meio de ações educativas que irão ensinar os estudantes a utilizarem o álcool com moderação. A intervenção não visa o confronto ou o julgamento sobre o comportamento de consumo; pelo contrário, visa à compreensão e à orientação direcionadas para promover a redução do consumo e das consequências negativas, utilizando os princípios da entrevista motivacional e de estratégias cognitivocomportamentais específicas.

O BASICS é caracterizado como um programa de baixo custo e de fácil aceitação por parte dos estudantes, envolvendo apenas duas sessões de 50 minutos de atendimento individual (Dimeff et al., 2002). Marques e Furtado (2004) e Pereira et al. (2013) realizaram um estudo de revisão bibliográfica sobre a efetividade de intervenções breves para o uso abusivo do álcool e identificaram que esse tipo de estratégia geralmente é efetiva na redução da frequência e quantidade de consumo de álcool entre estudantes universitários, causando impacto positivo e imediato.

Ainda assim, identificam-se muito mais estudos internacionais sobre o BASICS, os quais discutem e comprovam a eficácia dessa intervenção, sendo que no Brasil esses estudos ainda são escassos. Portanto, este estudo teve como objetivo avaliar o padrão de consumo de álcool e suas consequências, antes e após a aplicação da intervenção BASICS, em uma amostra de estudantes universitários brasileiros.

\section{Método}

Realizou-se um estudo descritivo, quantitativo e de delineamento longitudinal entre janeiro de 2013 e maio de 2014.

\section{Participantes}

Participaram deste estudo 32 estudantes universitários, maiores de 18 anos e de ambos os gêneros, sendo em sua maioria estudantes do gênero feminino $(53,1 \%)$, da faixa etária de 18 a 24 anos $(84,4 \%)$ e com média de idade de 22,53 anos $(D P=3,61)$, todos da Universidade Federal de São Paulo (UNIFESP) - Campus Diadema, sendo esses dos cursos de graduação de Ciências Biológicas, Ciências Ambientais, Farmácia e Bioquímica, Área de Química (Química, Engenharia Química e Química Industrial) e Ciências, do $3^{\circ}, 4^{\circ}$ ou $5^{\circ}$ ano. A amostra foi realizada por conveniência e de acordo com a aceitação do estudante em participar da pesquisa.

\section{Instrumentos}

Foi utilizado para a coleta de dados um questionário autoaplicável composto por três instrumentos, que foram:

The Alcohol Use Disorders Identification Test (AUDIT) desenvolvido pela Organização Mundial da Saúde (Babor, Higgins-Biddle, Saunders, \& Monteiro, 2001) e validado para a população brasileira por Lima et al. (1999). Tem como objetivo identificar padrões de consumo de álcool, como uso de baixo risco ( 0 a 7 pontos), uso de risco (8 a 15 pontos), uso nocivo (16 a 19 pontos) ou provável dependência (acima de 20 pontos).

Rutgers Alcohol Problem Index (RAPI) desenvolvido por White e Labouvie (1989), tem como objetivo avaliar problemas relacionados ao consumo de álcool, indicando as consequências negativas associadas ao uso. Esse instrumento apresenta propriedades psicométricas confiáveis e adequadas ao objetivo que se propõe, sendo que já foi traduzido para diversas línguas, inclusive para o português falado no Brasil (Dimeff et al., 2002; White \& Labouvie, 1989).

O RAPI é um instrumento constituído por 23 itens, os quais devem ser respondidos considerando uma escala de 0 a 4 , sendo $0=$ nunca, $1=$ uma a duas vezes, $2=$ três a cinco vezes, 3 = seis a dez vezes e $4=$ mais que dez vezes, sendo que cada item deve ser respondido levando em consideração os últimos 12 meses. As respostas irão indicar o 
número de vezes em que um dado comportamento ocorreu em decorrência do consumo de bebidas alcóolicas (Dimeff et al., 2002; White \& Labouvie, 1989).

Questionário de Caracterização dos Estudantes desenvolvido pelos autores do presente estudo, para levantamento de dados sociodemográficos dos estudantes. O estudo ainda incluiu o Critério de Classificação Econômica Brasil (CCEB), elaborado pela Associação Brasileira de Empresas de Pesquisa (ABEP, 2013). O CCEB é um questionário que avalia classes econômicas (A1, A2, B1, B2, C1, C2, D e E) a partir da presença de bens de consumo e do grau de instrução do chefe da família (ABEP, 2013).

\section{Procedimentos}

Após a autorização da direção da universidade, aplicou-se em sala de aula os instrumentos. O AUDIT foi utilizado para triar estudantes que realizam uso de risco, nocivo ou com provável dependência de álcool. Segundo dados da Pró-Reitoria de Graduação, existiam, aproximadamente, 2.250 estudantes matriculados no ano de 2012 neste Campus. No entanto, nos dias da aplicação do questionário, em sala de aula, haviam 794 estudantes, sendo que 786 questionários foram totalmente preenchidos e considerados para o presente estudo.

Do total de 786 estudantes inicialmente avaliados, 135 foram identificados com padrão de uso de álcool caracterizados por uso de risco, nocivo ou com provável dependência, e estes foram convidados por meio de $e$ -mail a participar da segunda etapa da pesquisa. Dos 135 estudantes inicialmente identificados, 42 concordaram em participar do grupo controle ou do grupo experimental (intervenção) e foram distribuídos aleatoriamente. Três estudantes desistiram e não compareceram ao segundo encontro da intervenção. Desses 39 estudantes, quatro não foram localizados no momento da primeira reavaliação, realizada seis meses após a intervenção e, outros três, não foram localizados no momento da segunda reavaliação, realizada após um ano da intervenção. Assim, participaram do presente estudo, 32 estudantes que concluíram todas as etapas propostas.

Com a finalidade de se avaliar os efeitos da intervenção, os estudantes foram separados aleatoriamente em dois grupos, um experimental e outro controle, sendo que os estudantes do grupo controle, após o término de todas as reavaliações, foram convidados a participar da intervenção por razões éticas.

\section{Intervenção Breve}

A intervenção breve BASICS consiste em uma intervenção de dois encontros com intervalo de 15 dias e com duração de até 50 minutos cada. No primeiro encontro são coletadas informações a respeito do motivo pelo qual o estudante consome bebidas alcóolicas e como aprendeu a consumir, suas crenças e expectativas em relação ao álcool e informações específicas sobre o consumo, tais como: tipo de bebida mais consumida, frequência de consumo, quantidade, locais mais frequentes de consumo, se acom- panhado ou não e histórico familiar de consumo de álcool (Dimeff et al., 2002).

Ao final do primeiro encontro foi solicitado que o estudante preenchesse o RAPI e foi entregue a ele um instrumento (Daily Drinking Questionnaire) para que anotasse informações sobre o seu consumo de álcool durante os próximos 15 dias, devendo anotar a bebida consumida; a quantidade, em doses; em quantas horas consumiu essa quantidade; em que local estava e se estava acompanhado ou não (Dimeff et al., 2002).

O segundo encontro com o estudante, denominado de retroalimentação pelos autores da versão brasileira do BASICS (Dimeff et al., 2002), tem o objetivo de informar ao estudante sobre seu consumo de álcool, assim como orientá-lo a como beber de forma que isto não afete sua saúde física e mental. Neste sentido, são passadas informações, por meio de um material educativo, acerca do seu padrão de consumo, se é de risco, nocivo ou se apresenta indicativos de dependência; seus gastos financeiros com álcool; calorias consumidas; comparação com o consumo dos demais estudantes e as possíveis consequências que esse padrão de consumo já apresenta em sua vida.

Além disso, são transmitidas informações sobre riscos à saúde, benefícios da moderação e seus prós e contras, estratégias de uso moderado de álcool, efeitos do nível de álcool no sangue e alguns mitos e curiosidades a respeito do álcool. Todas essas informações contidas no livro de Dimeff et al. (2002) foram sintetizadas e utilizadas para compor um material educativo, composto por nove páginas, como um feedback personalizado, que foi trabalhado e entregue ao estudante durante o segundo encontro da intervenção.

\section{Análise dos Dados}

Para tabulação dos dados foi utilizado o programa estatístico R versão 3.0.1 (R Core Team, 2013). A análise descritiva das variáveis abrangeu frequência, porcentagem, média e desvio padrão. Para comparações entre o grupo controle e o grupo experimental nos três momentos de aplicação (inicial, após seis meses e após um ano) dos instrumentos AUDIT e RAPI foi utilizado o modelo de análise de variância com medidas repetidas e, posteriormente, $o$ método de comparações múltiplas de Bonferroni. O nível de significância adotado foi de $p<0,05$.

\section{Considerações Éticas}

Este estudo foi submetido ao Comitê de Ética em Pesquisa da UNIFESP (CAAE: 08723112.8.0000.5505). Seguiu os princípios éticos contidos na Declaração de Helsinki e todos os participantes assinaram um Termo de Consentimento Livre e Esclarecido, autorizando sua participação e divulgação científica dos dados.

Cabe ressaltar, que no Termo de Consentimento Livre e Esclarecido os estudantes autorizaram o contato para convidá-los a participar da segunda etapa da pesquisa, caso atingissem um escore mínimo que já detectasse problema em relação à presença de risco no que se refere ao consumo de álcool. Dessa forma, todos os participantes que partici- 
Silva, E. C. \& Tucci, A. M. (2015). Intervenção Breve para Redução do Consumo de Álcool e suas Consequências em Estudantes Universitários Brasileiros.

param da segunda etapa (grupo controle e experimental) estavam conscientes que apresentavam problemas em relação ao seu consumo de álcool.

\section{Resultados}

A Tabela 1 apresenta as características sociodemográficas, hábito de fumar e prática de atividade física dos estudantes da amostra, separados por grupo (controle e ex- perimental). Observa-se uma predominância de estudantes na faixa etária de 18 a 24 anos $(84,4 \%)$, solteiros $(96,9 \%)$ e que não possuem uma religião $(56,2 \%)$. A maioria dos estudantes, ou seja, 53,1\% pertence a classe B1 (renda média bruta familiar de R\$ 5.241) ou B2 (renda média bruta familiar de $\mathrm{R} \$ 2.654$ ), 54,8\% residem com familiares e $87,1 \%$ não apresentam o hábito de fumar tabaco.

Importante considerar que não houve diferença significativa nas características sociodemográficas entre os grupos, sendo as mesmas muito similares.

Tabela 1

Caracterização Sociodemográfica, Hábito de Fumar e Prática de Atividade Física dos Estudantes por Grupo

$\begin{array}{ccc}\text { Grupo Controle } & \text { Grupo Experimental } & \text { Total } \\ n=16(50 \%) & n=16(50 \%) & N=32(100 \%)\end{array}$

Faixa etária

18 a 24 anos

Mais de 25 anos

Sexo

Masculino

$8(50 \%)$

Feminino

$8(50 \%)$

$15(93,8 \%)$

$1(6,2 \%)$

$9(56,2 \%)$

$0(0 \%)$

$7(43,8 \%)$

$9(56,2 \%)$

$7(43,8 \%)$

Não

Prática de atividade física

Não pratica

$7(43,8 \%)$

$8(50 \%)$

Esporadicamente

$1(6,2 \%)$

Diariamente

Classe econômica
A
$2(12,5 \%)$
B
$8(50 \%)$
C
$6(37,5 \%)$
$\mathrm{D} / \mathrm{E}$
$0(0 \%)$

Fumante

Sim

$1(6,7 \%)$

$14(93,3 \%)$

Não
$14(87,5 \%)$

$27(84,4 \%)$

$2(12,5 \%)$

$5(15,6 \%)$

$7(43,8 \%)$

$15(46,9 \%)$

$9(56,2 \%)$

$17(53,1 \%)$

$16(100 \%)$

$31(96,9 \%)$

$0(0 \%)$

$1(3,1 \%)$

$8(53,3 \%)$

17 (54,8\%)

$1(6,7 \%)$

$1(3,3 \%)$

$6(40 \%)$

$13(41,9 \%)$

$5(31,2 \%)$

$14(43,8 \%)$

$11(68,8 \%)$

$18(56,2 \%)$

$7(43,8 \%)$

$14(43,8 \%)$

$8(50 \%)$

$16(50 \%)$

$1(6,2 \%)$

$2(6,2 \%)$

$2(12,5 \%)$

$4(12,5 \%)$

$9(56,3 \%)$

$17(53,1 \%)$

$4(25 \%)$

$10(31,3 \%)$

$1(6,2 \%)$

$1(3,1 \%)$

$3(18,8 \%)$

$4(12,9 \%)$

$13(81,2 \%)$

$27(87,1 \%)$ 
O padrão inicial de consumo de álcool dos grupos controle e experimental se mostrou similar, como pode ser observado na Tabela 2. A Tabela 2 mostra também que após a intervenção houve redução da frequência de estudantes nos níveis mais problemáticos de consumo de álcool em ambos os grupos, consequentemente, estudantes que se enquadravam no uso de risco ou nocivo passaram para um consumo de baixo risco. Após um ano da intervenção, metade dos estudantes de ambos os grupos $(N=16)$ já apresentavam um consumo de baixo risco.

Vale ressaltar que apenas um estudante foi classificado com provável dependência de álcool no momento inicial de avaliação. Para esse estudante, além da participação na segunda etapa da pesquisa, houve orientação e encaminhamento para atendimento especializado.

Tabela 2

Distribuição dos Grupos de acordo com o AUDIT e os Momentos de Avaliação

AUDIT $N=32(100 \%)$

\begin{tabular}{lccc}
\cline { 2 - 3 } & Avaliação inicial & Após 6 meses & Após 1 ano \\
\hline Uso de baixo risco & $0(0 \%)$ & $9(56,3 \%)$ & $9(56,3 \%)$ \\
$\quad$ Grupo Controle & $0(0 \%)$ & $6(37,4 \%)$ & $7(43,8 \%)$ \\
$\quad$ Grupo Experimental & & & \\
Uso de risco & $14(87,4 \%)$ & $6(37,5 \%)$ & $5(31,3 \%)$ \\
$\quad$ Grupo Controle & $14(87,5 \%)$ & $9(56,3 \%)$ & $8(50 \%)$ \\
$\quad$ Grupo Experimental & & & $2(12,4 \%)$ \\
Uso nocivo & $1(6,3 \%)$ & $0(0 \%)$ & $1(6,2 \%)$ \\
$\quad$ Grupo Controle & $2(12,5 \%)$ & $1(6,3 \%)$ & $0(0 \%)$ \\
$\quad$ Grupo Experimental & & $1(6,2 \%)$ & $0(0 \%)$ \\
Provável dependência & $1(6,3 \%)$ & $0(0 \%)$ & \\
$\quad$ Grupo Controle & $0(0 \%)$ & & \\
$\quad$ Grupo Experimental & & & \\
\hline
\end{tabular}

Observou-se, por meio da análise das questões do AUDIT, que uma maior frequência de estudantes passou a consumir menor quantidade de doses de bebidas alcoólicas. Antes da intervenção, o consumo de álcool dos estudantes de ambos os grupos (controle e experimental) em um dia típico era majoritariamente de 7 a 10 doses $(34,4 \%)$ ou mais de 10 doses $(18,8 \%)$ por ocasião, enquanto $15,6 \%$ dos estudantes consumiam de 3 a 4 doses e $31,2 \%$, de 5 a 6 doses. Após um ano, a minoria referiu beber de 7 a 10 doses $(15,6 \%)$ ou mais de 10 doses $(3,1 \%)$, enquanto $15,6 \%$ referiram beber até 2 doses; $31,3 \%$ de 3 a 4 doses e $34,4 \%$ de 5 a 6 doses por ocasião. Além disso, nas avaliações após seis meses e após um ano da intervenção, uma porcentagem significativa dos estudantes diminuiu a frequência com que consumiram 6 ou mais doses de álcool em uma única ocasião, considerado como prática de binge drinking. Cabe apontar que $12,5 \%$ deixaram de consumir em binge drinking nesse período, sendo que antes da intervenção todos os estudantes relataram prática de binge drinking.

Com relação às consequências decorrentes do consumo de álcool, notou-se que mais estudantes não deixaram de fazer algo que era esperado devido ao consumo de bebidas alcoólicas após a intervenção $(71,9 \%)$ quando comparados ao momento inicial $(43,8 \%)$. A mesma redução se deu com sentir-se culpado após o consumo de álcool. Inicialmente, $31,3 \%$ dos estudantes relataram que não se sentiam culpados após o consumo de bebidas alcoólicas; após um ano da intervenção, $53,1 \%$ referiram ter se sentido assim, demostrando uma sensibilização e conscientização dos estudantes para os efeitos maléficos de seu consumo de álcool.

Outro aspecto observado foi o fato do estudante relatar quantas vezes não conseguiu se lembrar do que havia ocorrido na noite anterior devido ao consumo de álcool. No primeiro momento, $90,6 \%$ dos estudantes relataram que isso aconteceu; após um ano da intervenção, esse índice foi de $54,1 \%$, demonstrando uma diminuição das consequências negativas decorrentes do consumo de álcool.

Observando as Figuras 1 e 2 é possível notar uma redução nas médias dos escores do AUDIT (Figura 1) e do RAPI (Figura 2) ao longo do tempo em que ocorreram as avaliações, tanto no grupo controle quanto no experimental. Não foi detectada diferença significativa entre os grupos controle e experimental ao longo dos três momentos das avaliações. 
Silva, E. C. \& Tucci, A. M. (2015). Intervenção Breve para Redução do Consumo de Álcool e suas Consequências em Estudantes Universitários Brasileiros.

A Figura 1 mostra as médias do AUDIT e revela que, no grupo controle houve redução significativa entre o primeiro (média $=11,3 ; D P=3,4)$ e o segundo momento (média $=8,44 ; D P=4,57)$ de avaliação $(p=0,001)$ e entre o primeiro e o terceiro momento (média $=8,50 ; D P=3,69)$ de avaliação $(p=0,001)$. Já, no grupo experimental houve redução significativa na média do AUDIT entre o momento inicial (média $=11,44 ; D P=3,37$ ) e o segundo momento (média $=8,81 ; D P=4,23)$ de avaliação $(p=0,001)$ e entre o primeiro e o terceiro momento de avaliação (média $=8,63$; $D P=4,06 ; p=0,001)$.

A Figura 1 indica que as médias do AUDIT, nos grupos controle e experimental, tiveram redução significativa entre o primeiro e o segundo momento de avaliação $(p=0,001)$ e entre o primeiro e o terceiro momento de avaliação $(p=0,001)$.

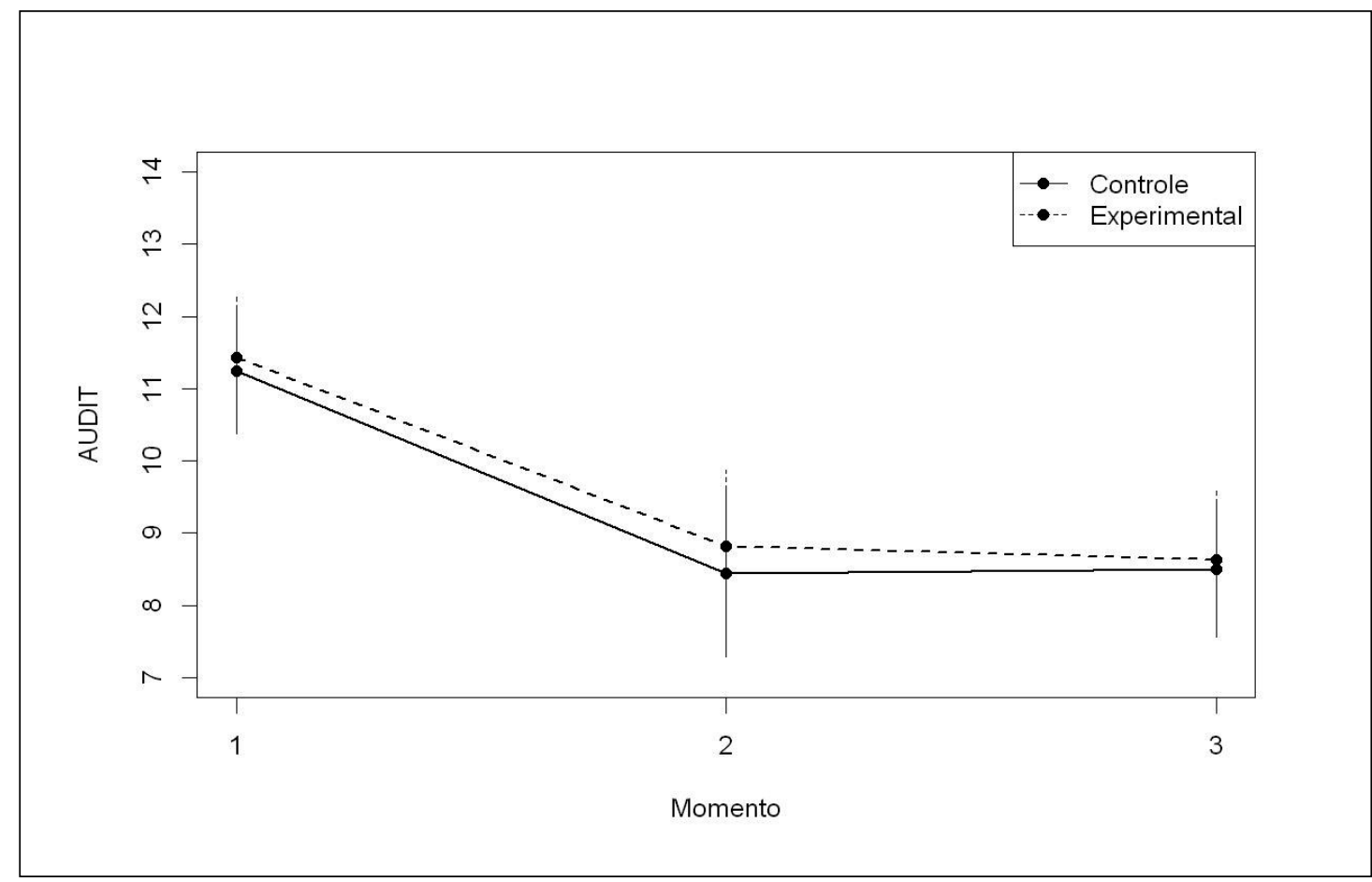

Figura 1. Escore médio da variável AUDIT de acordo com o grupo (controle e experimental) e o momento de avaliação.

A Figura 2 mostra as médias do RAPI e revela que, no grupo controle houve redução entre o primeiro (média $=11,25 ; D P=8,28)$ e o segundo momento (média $=6,63 ; D P=4,83)$ de avaliação $(p=0,003)$ e entre o primeiro e o terceiro momento (média $=8,13 ; D P=10,26$ ) de avaliação $(p=0,003)$. Já, no grupo experimental foi encontrada diferença significativa entre o primeiro (mé$\mathrm{dia}=8,50 ; D P=5,84$ ) e o segundo momento (média $=5,06$;
$D P=5,93)$ de avaliação $(p=0,003)$ e, entre o primeiro e o terceiro momento (média $=8,13 ; D P=10,26$ ) de avaliação $(p=0,003)$.

A Figura 2 indica que as médias do RAPI, nos grupos controle e experimental, tiveram redução significativa entre o primeiro e o segundo momento de avaliação $(p=0,003)$ e entre o primeiro e o terceiro momento de avaliação $(\mathrm{p}=0,003)$ 


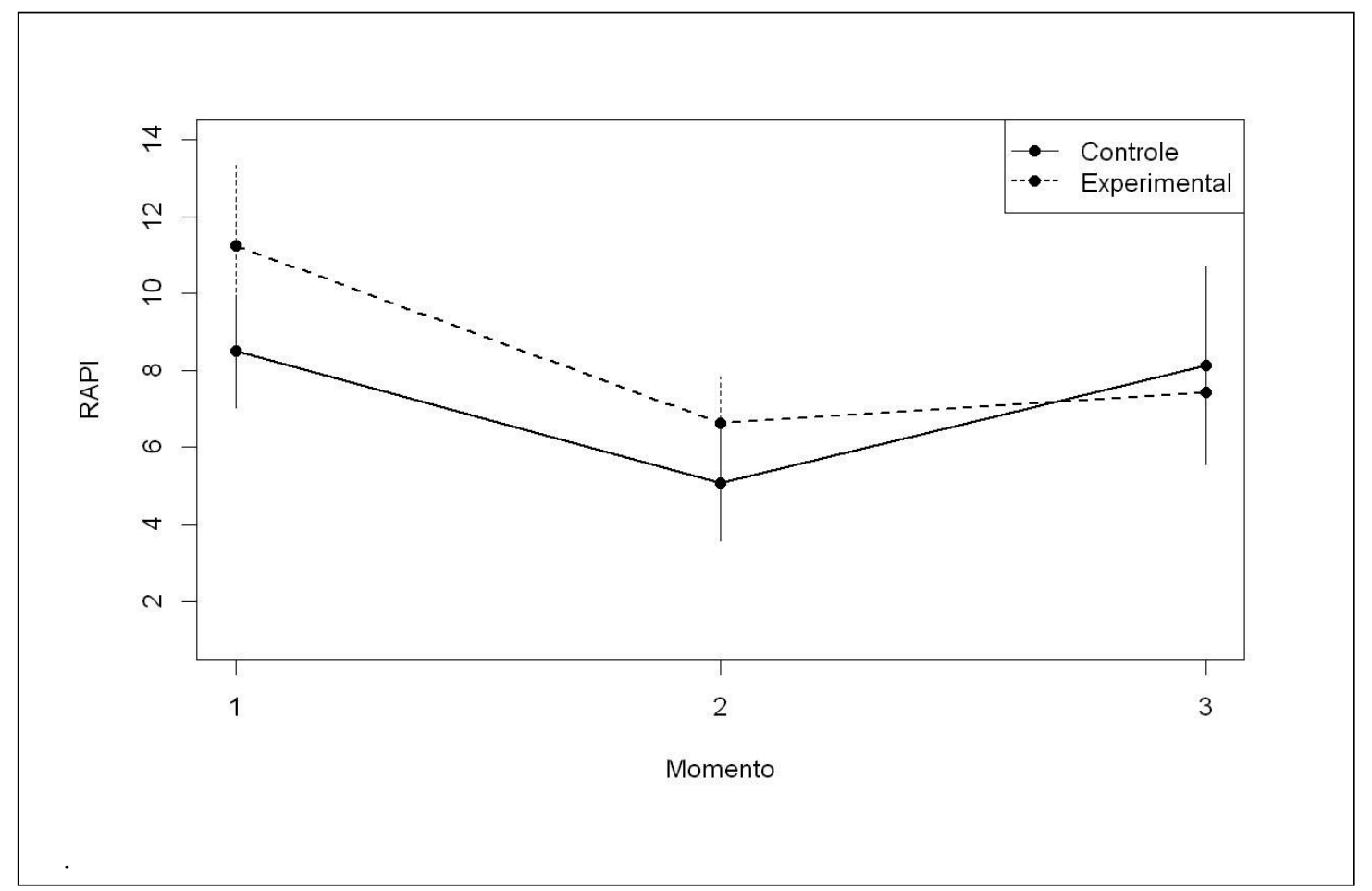

Figura 2. Escore médio da variável RAPI de acordo com o grupo e o momento de avaliação.

\section{Discussão}

Os estudantes universitários são considerados uma população de risco no que se refere ao consumo de bebidas alcoólicas, seja pela frequência de consumo ou por suas consequências, que inclusive podem afetar consideravelmente o desempenho acadêmico do estudante (Haas et al., 2012; Silva \& Petroski, 2012). Por essa razão, o consumo de álcool nessa população tem sido foco de preocupações e buscas de intervenções eficazes para diminuir o consumo, assim como os problemas e consequências associadas.

O presente estudo avaliou o padrão de consumo de álcool e suas consequências em estudantes de uma universidade federal brasileira, antes e após uma intervenção breve, o BASICS. Como já apontado na introdução, o BASICS é a única intervenção breve presente na literatura que foi desenvolvida e padronizada para o uso com estudantes universitários e, por essa razão, foi a escolhida para ser utilizada no presente estudo.

Os resultados deste estudo demonstram uma diminuição no consumo de álcool e das consequências associadas tanto no grupo controle como no grupo experimental. A redução do consumo de álcool em ambos os grupos também foi encontrada no estudo de Walters, Vader e Harris (2007), os quais realizaram uma intervenção breve com universitários de forma online. Estratégias de intervenções online estão crescendo cada vez mais e, apesar de haver poucos estudos na área, em curto prazo esse tipo de intervenção tem apresentado resultados favoráveis (Larimer, Cronce, Lee, \& Kilmer, 2005).
Por outro lado, os demais estudos que utilizaram a metodologia BASICS obtiveram resultados satisfatórios na redução da frequência do consumo de álcool e das consequências negativas associadas apenas no grupo experimental (Amaro et al., 2010; Borsari \& Carey, 2000; DiFulvio et al., 2012; Fachini, Aliane, Martinez, \& Furtado, 2012; Roberts, Kivlahan, Baer, Neal, \& Marlatt, 2000; Simão et al., 2008). No entanto, o estudo de Grossbard et al. (2010) encontrou diminuição significativa do consumo de álcool na frequência e quantidade consumida entre os estudantes do grupo experimental em comparação com o grupo controle, mas não encontrou diminuição significativa na redução das consequências negativas.

De maneira geral, os estudos que utilizaram em sua metodologia grupos controle e experimental têm identificado eficácia satisfatória da intervenção breve BASICS na diminuição do consumo e das consequências associadas no grupo experimental (Amaro et al., 2010; Borsari \& Carey, 2000; DiFulvio et al., 2012; Fachini et al., 2012), diferentemente dos dados do presente estudo. No entanto, pode-se considerar positivo e satisfatório o resultado do presente estudo visto que foi possível reduzir o consumo de álcool e as consequências negativas em ambos os grupos. Esses dados sugerem que pelo fato da pesquisa ser direcionada a essa temática pode ter pré-estabelecido um alerta para os riscos de um consumo abusivo de álcool para aqueles que apresentavam um padrão de consumo inicial mais problemático. Além disso, a partir da apresentação do Termo de Consentimento Livre e Esclarecido, os estudantes ficaram cientes que seriam chamados para a segunda 
Silva, E. C. \& Tucci, A. M. (2015). Intervenção Breve para Redução do Consumo de Álcool e suas Consequências em Estudantes Universitários Brasileiros.

etapa da pesquisa apenas aqueles que apresentavam um padrão de consumo de álcool problemático, acrescido do fato de terem tido tal conhecimento pelo e-mail enviado pela pesquisadora convidando-os para participarem da segunda etapa da pesquisa. Pode-se supor que tais conhecimentos podem ter proporcionado uma reflexão da maneira como consumiam álcool e, consequentemente, ter gerado modificação deste padrão sem mesmo terem passado pela intervenção.

Os estudos citados anteriormente não referiram se os estudantes dos grupos experimental e controle foram informados sobre o seu padrão de consumo ao serem convidados para participarem da intervenção BASICS, tão pouco se constava no Termo de Consentimento Livre e Esclarecido alguma informação que sugerisse que só os estudantes que consumiam de forma arriscada seriam convidados a participar de um grupo experimental ou controle. Dessa forma, não podemos afirmar que estes estudantes sabiam do seu padrão de consumo antes de participarem da intervenção (Amaro et al., 2010; Borsari \& Carey, 2000; DiFulvio et al., 2012; Fachini et al., 2012; Grossbard et al., 2010; Roberts et al., 2000; Simão et al., 2008), fato este que pode apontar para a diferença encontrada no presente estudo e os demais que utilizaram o mesmo método de intervenção aqui proposto.

Assim, os resultados do presente estudo apontam que a utilização de diferentes instrumentos para se identificar indivíduos que consomem álcool de forma arriscada e o posterior conhecimento pelos estudantes acerca desses resultados pode ser útil não apenas para identificação do padrão de consumo, mas para que haja conscientização do indivíduo sobre os possíveis riscos desse consumo, favorecendo a mudança no padrão de consumo de álcool. Os instrumentos que têm sido mais utilizados para este objetivo são: The Michigan Alcholism Screening Test (MAST), Self-Administered Alcohol Screenning Test (SAAST), Young Adult Alcohol Problem Screnning Test (YAAPST), T-ACE, TWEAK, CAGE, e o The Alcohol Use Disorders Identification Test (AUDIT) (HigginsBiddle \& Barbor, 2000; Mastroleo, Turrisi, Carney, Ray, \& Larimer, 2010).

A partir dos resultados deste estudo é possível considerar que o conhecimento dos estudantes sobre a existência de risco em relação ao seu consumo de álcool pode gerar efeitos de conscientização sobre o mesmo e alterar seu padrão de comportamento frente ao consumo de álcool. Portanto, os dados do presente estudo sugerem que apenas a aplicação de instrumentos específicos que avaliem o padrão de consumo de álcool e as consequências associadas, assim como a posterior disponibilização desses dados para os estudantes, pode ser efetivo em alterar o padrão de consumo de álcool entre estudantes universitários, sendo tal intervenção de baixo custo e podendo atingir um maior número de pessoas (HigginsBiddle \& Barbor, 2000).

\section{Conclusão}

Este estudo revelou diminuição do consumo de álcool e suas consequências negativas entre estudantes universitários tanto do grupo controle quanto do grupo experimental. Tal resultado indica uma importante contribuição na área e sugere que um simples levantamento do padrão de consumo de álcool a partir de instrumentos especializados e o respectivo feedback sobre este padrão pode gerar efeitos positivos na diminuição do consumo de álcool, assim como das suas consequências entre estudantes universitários. Assim, entende-se que ter o conhecimento acerca de um padrão de consumo de álcool de risco pode conscientizar os estudantes e gerar redução desse consumo, assim como das suas consequências.

Apesar deste estudo não ter encontrado diferença significativa entre os grupos controle e experimental, inviabilizando a avaliação da eficácia da intervenção breve BASICS, o mesmo apresentou dados importantes que podem ser discutidos para se repensar outras formas de intervenção, mais simples, de menor custo e que possam ter eficácia semelhante às intervenções mais complexas e mais elaboradas.

Portanto, os dados deste estudo não descaracterizam a importância das intervenções breves, apenas sugerem que a aplicação de instrumentos de screening e com feedback podem apresentar resultados também efetivos tanto quanto as intervenções breves para a diminuição do consumo de álcool entre estudantes universitários.

Apesar das contribuições deste estudo, este apresenta algumas limitações, principalmente com relação ao tamanho e especificidade da amostra, ou seja, por ser específica de um Campus do processo de expansão das universidades federais, o REUNI. Assim, tais resultados não podem ser generalizados para toda a população de estudantes universitários.

Ainda assim, sugere-se que outros estudos sejam realizados com a aplicação de questionários e testes de identificação do consumo de álcool e que seja fornecido feedback de forma rápida, para avaliar a eficácia desse tipo de intervenção, inclusive as realizadas de forma online, que poderão incluir um maior número de estudantes.

\section{Referências}

Amaro, H., Reed, E., Rowe, E., Picci, J., Mantella, P., \& Prado, G. (2010). Brief screening and intervention for alcohol and drug use in a college student health clinic: Feasibility, implementation, and outcomes. Journal of American College Health, 58(4), 357-364. doi:10.1080/07448480903501764

Andrade, A. G., Duarte, P. C. A. V., \& Oliveira, L. G. (Eds.). (2010). I Levantamento Nacional sobre o uso de álcool, tabaco e outras drogas entre universitários das 27 capitais brasileiras. Brasília, DF: Secretaria Nacional de Políticas sobre Drogas.

Associação Brasileira de Empresas de Pesquisa. (2013). Critério de classificação econômica Brasil. São Paulo, SP: Autor. 
Babor, T. F., Higgins-Biddle, J. C., Saunders, J. B., \& Monteiro, M. G. (2001). The Alcohol Use Disorders Identification Test: Guidelines for use in primary care. Geneva, Switzerland: World Health Organization.

Borsari, B., \& Carey, K. B. (2000). Effects of a brief motivational intervention with college students drinkers. Journal of Consulting Clinical Psychology, 68(4), 728-733. doi:10.1037/0022-006X.68.4.728

Cislaghi, J. F. (2010). Análise do Reuni: Uma nova expressão da contra-reforma universitária brasileira (Dissertação de mestrado em Serviço Social, Universidade do Estado do Rio de Janeiro, RJ, Brasil).

DiFulvio, G. T., Linowski, S. A., Mazziotti, J. S., \& Puleo, E. (2012). Effectiveness of the brief alcohol and screnning intervention of college students (BASICS) program with mandated population. Journal of American College Health, 60(4), 269-280. doi:10.1080/07448481.2011.599352

Dimeff, L. A., Baer, J. S., Kivlahan, D. R., \& Marlatt, G. A. (2002). Alcoolismo entre estudantes universitários: Uma abordagem de redução de danos. São Paulo, SP: Editora da Universidade Estadual Paulista "Júlio de Mesquita Filho".

Fachini, A., Aliane, P. P., Martinez, E. Z., \& Furtado, E. F. (2012). Efficacy of brief alcohol screening intervention for college students (BASICS): A meta-analysis of randomized controlled trials. Substance Abuse Treatment, Prevention, and Policy, 7(40), 1-10. doi:10.1186/1747-597X-7-40

Grossbard, J. R., Mastroleo, N. R., Kilmer, J. R., Lee, C. M., Turrisi, R., Larimer, M. E., \& Ray, A. (2010). Substance use patterns among first-year college students: Secondary effects of a combined alcohol intervention. Journal of Substance Abuse Treatment, 39(4), 384-390. doi:10.1016/j.jsat.2010.07.001

Haas, A. L., Smith, S. K., Kagan, K., \& Jacob, T. (2012). Pre-college pregaming: Practices, risk factors, and relationship to other indices of problematic drinking during the transition from high school to college. Psychology of Addictive Behaviors, 26(4), 931-938. doi:10.1037/a0029765

Higgins-Biddle, J. C., \& Babor, T. F. (2000). Alcohol screnning and brief intervention: Dissemination strategies for medical practice and public health. Addiction, 95(5), 677-686. doi:10.1046/j.1360-0443.2000.9556773.x

Larimer, M. E., Cronce, J. M., Lee, C. M., \& Kilmer, J. R. (2005). Brief intervention in college settings. Alcohol Research \& Health, 28(2), 94-104.

Lima, C. T., Freire, A. C., Silva, A. P., Teixeira, R. M., Farrell, M., \& Prince, M. (1999). Concurrent and construct validity of the AUDIT in a urban Brazilian sample. Alcohol \& Alcoholism, 40(6), 584-589. doi:10.1093/alcalc/agh202

Lorant, V., Nicaise, P., Soto, V. E., \& D'Hoore, W. (2013). Alcohol drinking among college students: College responsibility for personal troubles. BMC Public Health, 13(615), 1-9. doi:10.1186/1471-2458-13-615

Marques, A. C. P. R., \& Furtado, E. F. (2004). Intervenções breves para problemas relacionados ao álcool. Revista Brasileira de Psiquiatria, 26(1), 28-32. doi:10.1590/S151644462004000500008

Mastroleo, N. D., Turrisi, R., Carney, J. V., Ray, A. E., \& Larimer, M. E. (2010). Examination of post-training supervision of peer counselors in a motivational enhancement intervention to reduce drinking in a sample of heavy drinking college students. Journal of Substance Abuse Treatment, 39(3), 289-297. doi:10.1016/j.jsat.2010.06.00

Organização das Nações Unidas. (2013). United Nations Office for Drug Control and Crime Prevention - UNODCCP (World Drug Report No. E.13.XI.6). Vienna, Austria: Author.
Pedrosa, A. A. S., Camacho, L. A. B., Lambert, S. R., \& Oliveira, R. V. C. (2011). Consumo de álcool entre estudantes universitários. Cadernos de Saúde Pública, 27(8), 1611-1621. doi:10.1590/S0102-311X2011000800016

Pereira, M. O., Anginomi, B. M., Ferreira, N. C., Oliveira, M. A. F., Vargas, D., \& Colvero, L. A. (2013). Efetividade da intervenção breve para o uso abusivo de álcool na atenção primária: Revisão sistemática. Revista Brasileira de Enfermagem, 66(3), 420-428. doi:10.1590/S0034-71672013000300018

R Core Team. (2013). R: A language and environment for statistical computing [Computer software]. Vienna, Austria: R Foundation for Statistical Computing.

Ramis, T. R., Mielke, G. I., Habeyche, E. C., Oliz, M. M., Azevedo, M. R., \& Hallal, P. C. (2012). Tabagismo e consumo de álcool em estudantes universitários: Prevalência e fatores associados. Revista Brasileira de Epidemiologia, 15(2), 376385. doi:10.1590/S1415-790X2012000200015

Roberts, L. J., Kivlahan, D. R., Baer, J. S., Neal, D. J., \& Marlatt, G. A. (2000). Individual drinking changes following a brief intervention among college students: Clinical significance in an indicated preventive context. Journal of Consulting and Clinical Psychology, 68(3), 500-505. doi:10.1037/0022$-006 X .68 .3 .500$

Silva, D. A. S., \& Petroski, E. L. (2012). The simultaneous presence of health risk behaviors in freshman college students in Brasil. Journal of Community Health, 37(3), 591-598. doi:10.1007/s10900-011-9489-9

Simão, M. O., Kerr-Corrêa, F., Smaira, S. I., Trinca, L. A., Dalven, I., Martins, R. A., ...Tucci, A. M. (2008). Epidemiology and prevention. Prevention of "risky" drinking among students at a Brazilian university. Alcohol \& Alcoholism, 43(4), 470476. doi:10.1093/alcalc/agn019

Wagner, G. A., Oliveira, L. G., Barroso, L. P., Nishimura, R., Ishihara, L. M., \& Stempliuk, V. A. (2012). Drug use in college students: A 13-year trend. Revista de Saúde Pública 46(3), 497-504. doi:10.1590/S0034-89102012005000033

Walters, S. T., Vader, A. M., \& Harris, T. R. (2007). A controlled trial of web-based feedback for heavy drinking college students. Prevention Science, 8, 83-88. doi:10.1007/s11121006-0059-9

White, H. R., \& Labouvie, E. W. (1989). Towards the assessment of adolescent problem drinking. Journal of Studies on Alcohol, 50(1), 30-37. 